\title{
Solar Physics at the Einstein Tower
}

\author{
C. Denker ${ }^{1, \star}$, C. Heibel ${ }^{1,2}$, J. Rendtel $^{1}$, K. Arlt ${ }^{1}$, H. Balthasar ${ }^{1}$, A. Diercke ${ }^{1,3}$, \\ S. J. González Manrique ${ }^{1,3}$, A. Hofmann ${ }^{1}$, C. Kuckein ${ }^{1}$, H. Önel ${ }^{1}$, V. Senthamizh Pavai ${ }^{1,3}$, \\ J. Staude ${ }^{1}$, and M. Verma ${ }^{1}$ \\ 1 Leibniz-Institut für Astrophysik Potsdam (AIP), An der Sternwarte 16, 14482 Potsdam, Germany \\ 2 Humboldt-Universität zu Berlin, Unter den Linden 6, 10099 Berlin, Germany \\ 3 Universität Potsdam, Institut für Physik and Astronomie, Karl-Liebknecht-Straße 24/25, 14476 Potsdam, Germany
}

Received 23 September 2016, accepted XXXX

Published online XXXX

Key words history and philosophy of astronomy - Sun: photosphere - Sun: magnetic fields - techniques: spectroscopic - telescopes

The solar observatory Einstein Tower (Einsteinturm) at the Telegrafenberg in Potsdam is both a landmark of modern architecture and an important place for solar physics. Originally built for high-resolution spectroscopy and measuring the gravitational redshift, research shifted over the years to understanding the active Sun and its magnetic field. Nowadays, telescope and spectrographs are used for research and development, i.e., testing instruments and in particular polarization optics for advanced instrumentation deployed at major European and international astronomical and solar telescopes. In addition, the Einstein Tower is used for educating and training of the next generation astrophysicists as well as for education and public outreach activities directed at the general public. This article comments on the observatory's unique architecture and the challenges of maintaining and conserving the building. It describes in detail the characteristics of telescope, spectrographs, and imagers; it portrays some of the research and development activities.

Copyright line will be provided by the publisher

\section{Introduction}

The $12^{\text {th }}$ Potsdam Thinkshop in October 2015 was dedicated to the theme "The Dynamic Sun - Exploring the Many Facets of Solar Eruptive Events". The Einstein Tower served as the background image of the conference poster (Fig. 1), and the meeting concluded with a visit to the Einstein Tower highlighting the major achievements of this observatory, its remarkable architecture, and its contemporary use as a laboratory for instrument development and as a place for training the next generation of astrophysicists. The great interest expressed by the participants motivated us to write this article from the perspective of scientists who were and still are active at the solar observatory Einstein Tower.

The Astrophysical Observatory Potsdam (AOP) was founded in 1874 as the first observatory carrying the term astrophysics in its name (Staude 1991). This marks the transition from astronomy focused on external characteristics of celestial objects to astrophysics as a theory-driven science exploring the physical nature of these bodies. The Telegrafenberg in Potsdam was named after the Prussian semaphore system (built in 1832) for optical communication between Berlin and Koblenz. Besides AOP, the historical science park comprises several institutes such as the Magnetic Observatory (1889), the Meteorological Observatory (1890), and the Geodetic Institute (1892). A location

^ Corresponding author: cdenker@aip.de to the South of the Great Refractor (1899) was chosen as the construction site of the Einstein Tower, where erection of the tower started in the summer of 1920, finishing the shell construction already one year later. The construction faced economically challenging times (postwar reconstruction and hyperinflation). Interior construction and outfitting of the laboratory and work spaces commenced in 1922/23. The telescope optics and its support structure were manufactured by Carl Zeiss in Jena starting in 1919. The telescope was installed in 1924, and first observations were carried out on 1924 December 6 (Jäger 1986). The Einstein Tower thus complemented the broad spectrum of research concentrated on the Telegrafenberg, and in particular continued and expanded solar physics research, which was always an integral part of AOP's astrophysical research program (Staude 1995). A detailed description of the architectural achievements and of the historical background is presented in the monographs by Eggers et al. (1995) and Wilderotter (2005).

The Einstein Tower is a unique landmark of expressionist, utopian, and symbolic architecture at the beginning of the $20^{\text {th }}$ century - initiated by the astronomer Erwin Finlay Freundlich $\left({ }^{*} 1885-{ }^{+} 1964\right)$, designed by the architect Erich Mendelsohn $\left({ }^{*} 1887-{ }^{\dagger} 1953\right)$, and supported by Albert Einstein $\left({ }^{*} 1879-+1955\right)$. Their interaction was driven by the ambition to find observational proof of the general theory of relativity. This endeavor also received support by Karl Schwarzschild $\left({ }^{*} 1873-{ }^{+} 1916\right)$, director of AOP, who him- 


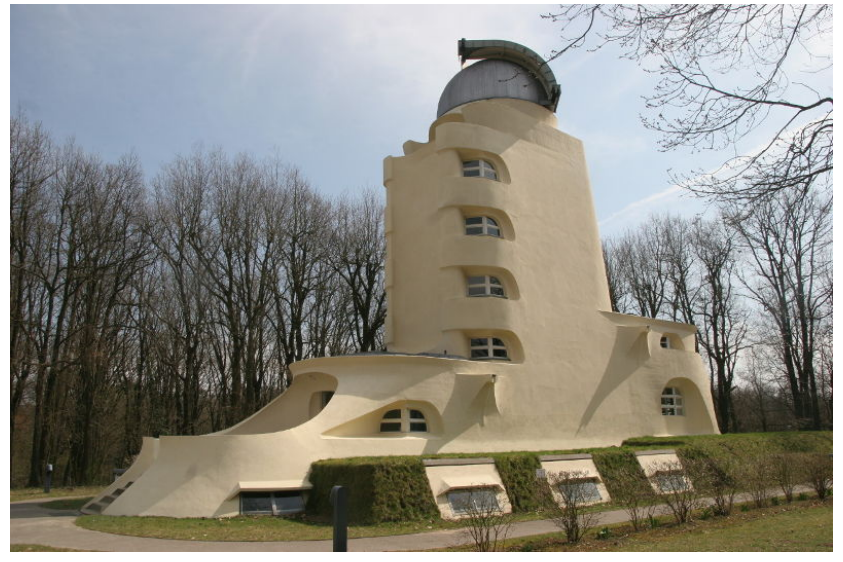

Fig. 1 North-west view of the Einstein Tower in its original light yellow-ochre color scheme. The dome is opened for solar observations. The grass covered basement contains optical laboratory, spectrograph chamber, and workshops.

self contributed to the general theory of relativity with the first exact solutions to the field equations. This intriguing paradigm shift from astronomy to astrophysics, marking a new era of physics - the confluence of quantum theory and relativity, was explored by Hentschel (1992) in his monograph from the perspective of the history of science.

Einstein released a preliminary version of his general theory of relativity in 1911, which expands the special theory of relativity to include accelerated frames of reference and the equivalence principle of gravitational and inertial mass. He pointed out three effects that are in principle accessible to astronomical observations (Jäger 1986): (1) rotation of apsidal nodes of planetary orbits (known since about 1850 for Mercury), (2) bending of light when passing heavy objects such as stars and galaxies, and (3) the gravitational redshift. The confirmation of the second effect, during an eclipse expedition by Sir Arthur Eddington and Frank Watson Dyson in 1919, was the starting signal and impetus to raise funding for the Einstein Tower. The new solar observatory was financed in equal parts by the state of Prussia and the Albert Einstein Foundation of the German Industry. The foundation's board of trustees included among others Einstein as the chairman, Freundlich as the head of the institute, and the director of AOP. The board convened regularly in the meeting room of the Einstein Tower, which was outfitted with furniture also designed by Mendelsohn (Fig. 2). Board meetings were concerned with the operation of the Einstein Tower, i.e., finances, acquisition of new instruments, and recurrent structural damages to the building.

Freundlich as head of the Einstein Institute was in charge of the Einstein Tower from 1920 to 1933. With the take-over of power by the National Socialist Party in 1933, Freundlich emigrated to Turkey and Einstein's name was removed from the institute by renaming it into Institute for Solar Physics, and soon thereafter the observatory was absorbed and integrated in AOP. The monography by Sailer (2007) recounts the history of solar research in Germany

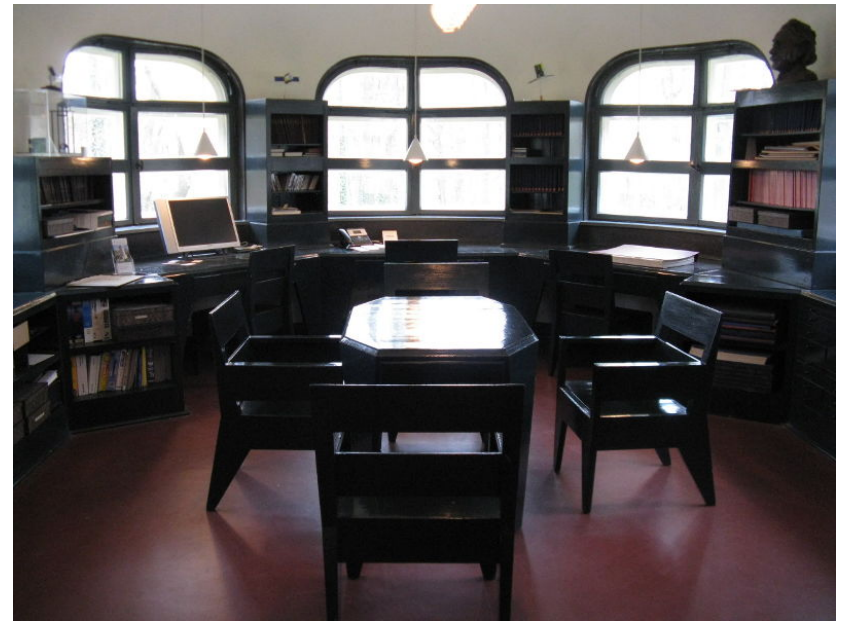

Fig. 2 A small library and the photographic plate archive of the Einstein Tower are contained in the meeting room on the first floor. Composition of interior walls and furnishing were conceptualized in its entirety by Erich Mendelsohn, who also designed the furniture.

from 1939-1949 and its entanglement with the German Luftwaffe. After World War II, the Einstein Tower belonged to the German Academy of Sciences (in 1946), the Central Institute for Solar-Terrestrial Physics/Heinrich Hertz Institute (in 1967), and the Central Institute for Astrophysics (in 1983). Following the German reunification, the solar observatory became part of the Astrophysical Institute Potsdam (1992), which was renamed to Leibniz Institute for Astrophysics Potsdam (AIP) in 2011.

\section{Preserving the Einstein Tower}

The mixed construction of the Einstein Tower consisting of bricks, concrete, and rendering is the underlying cause for a recurring damage pattern. The concrete quality and iron reinforcements do not conform to current standards, and no efficient way to remove rain water and humidity was available leading to fissures in the rendering coat, which facilitate an easy entry of rain water and moisture. This caused damages after freezing temperatures and corrosion of the iron reinforcements (structural members) above windows and throughout the building structure (Pitz \& Hoh 1996).

The first major repairs in 1927 because of widespread moisture penetration commenced under the supervision of Mendelsohn himself leading to a massive addition of sheet metal (window sills and roofing) significantly changing the character of the building. These measures, however, were not very effective necessitating major repairs about every 5-10 years. Regrettably, the history of the Einstein Tower is also a history of recurring damages and endless renovations. The second major overhaul in 1940/41 was driven by fungal infestation that damaged the prism spectrograph in 1937. Towards the end of World War II, a blockbuster detonation during an air-raid in proximity to the Ein- 

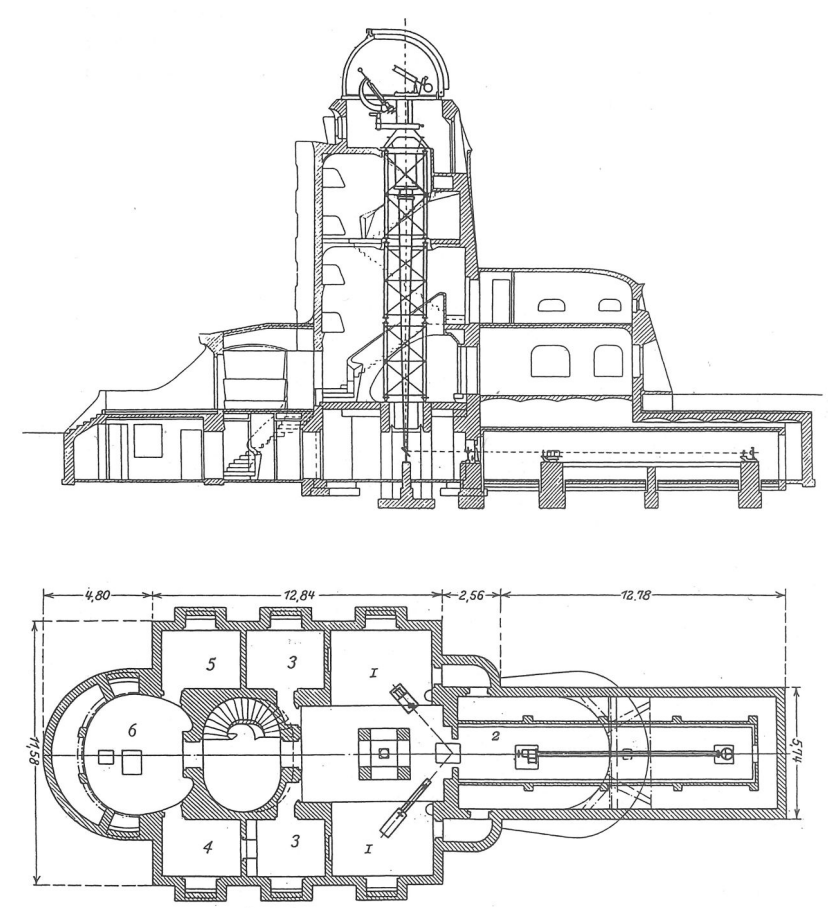

Fig. 3 Vertical section of the Einstein Tower (top) and horizontal section of its basement (bottom) with the historical floor plan: (1) laboratory with turning mirror, (2) thermally stabilized spectrograph chamber, (3) workshops, (4) dark room, (5) high-voltage battery, and (6) microphotometer room (see Fig. 2 in Freundlich 1927).

stein Tower severely damaged entrance, dome, windows, doors, and even interior walls. However, immediate repairs in September 1946 allowed solar observations to continue soon thereafter. Major repairs of the building followed in 1950, 1958, 1964, 1974-1978, and 1984. By the beginning of the 1990ies the condition of the Einstein Tower deteriorated to the point, where the continued existence of the building was endangered (Pitz \& Hoh 1996).

Intensive research into the origin and causes of the recurring damage pattern preceded the latest major repair, which was made possible by generous support of the Wüstenrot Foundation (covering two-thirds of about 1.5 Million Euros) and was completed in 1999. The restoration was carried out in close collaboration with State Office for Monument Protection (Landesamt für Denkmalpflege) and included: renewal of the rendering coat conserving the original rendering whenever possible, return to an ochre-colored coat of paint for the entire facade, removal of the ineffective sheet metal installed in 1927, returning the dominant entrance terrace to its original appearance, and elimination of dampness and moisture damage in the basement. The guiding principles of the renovation and restoration were to preserve the artistic and corporeal substance of the Einstein Tower as much as possible, to facilitate easy access and procedures for future repairs, and to safeguard the building while upholding its function as a place for astrophysical re-

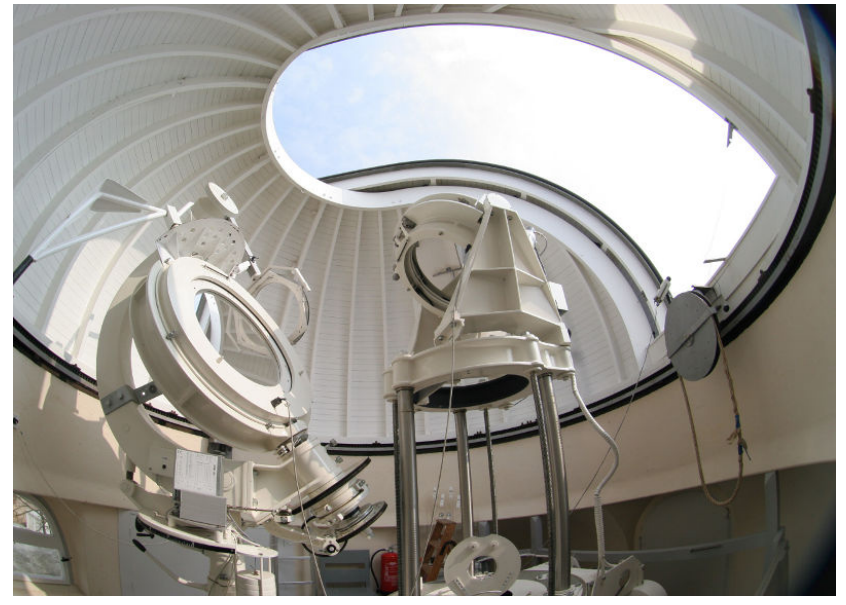

Fig. 4 Coelostat system inside the metal-plated wooden dome. The dome rotation is motorized but the shutter has to be opened manually. The boom over the first coelostat mirror carries a large optical mount for polarization calibration. A mechanism to change the telescope aperture is located below the second coelostat mirror, which sends the light vertically into the tower.

search. At the end of the renovation it became evident that continuous monitoring and repairs will be required in the future (Pitz \& Hoh 2006a). This led to a long-term conservation plan (Pitz \& Hoh 2006b) because causes for damage could not be completely removed but only mitigation plans were put into place.

A fire during the restoration of the Einstein Tower on 1998 January 6 led to significant damages to the wooden support structure and the telescope itself. The mechanical mounts of the coelostat mirrors and the entrance aperture were cleaned of soot and other remnants of the fire. Cleaning of the $60-\mathrm{cm}$ doublet lens required a careful evaluation of potential surface damages, which fortunately had not occurred, so that after a special cleaning procedure, the lens could be restored to its previous performance. On 1999 July 27, the Einstein Tower resumed operations (Balthasar $\&$ Artus 2000).

In the 2015 competition of the Getty Foundation's "Keeping It Modern" initiative the Einstein Tower received a planing and conservation grant. Several major tasks are currently carried out supported by this grant: (1) validate that the cyclic maintenance plan is effective and sustainable, (2) identify an efficient solution to prevent dampness and moisture damage in the subterranean laboratory, which houses the high-resolution spectrograph with its sensitive optics and gratings, (3) install a better heating and temperature control system for the basement using modern, energy efficient systems, and (4) investigate the thermally induced stresses in the hull of the Einstein Tower using simulations to clearly identify weaknesses in the building structure, to reveal hidden causes for damages, and to guide future mitigation strategies. 


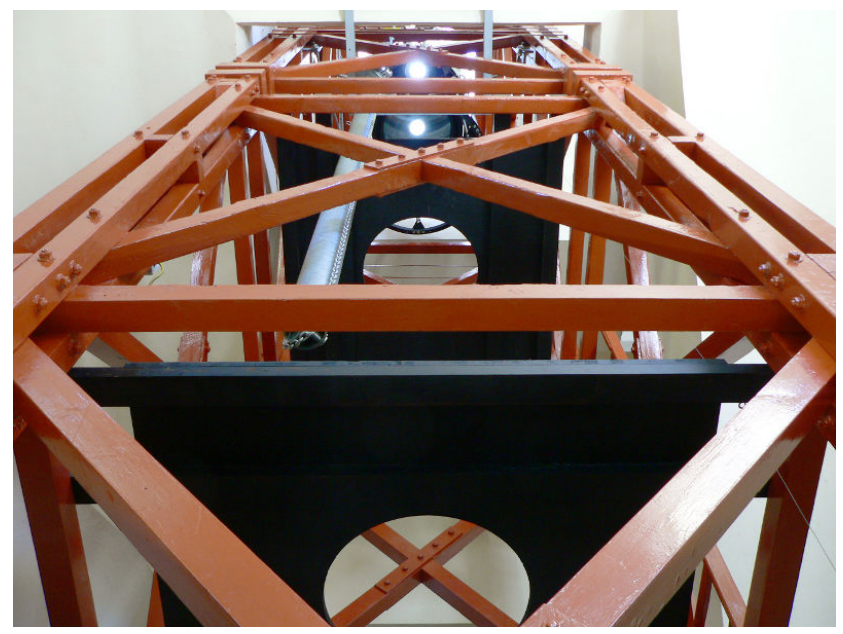

Fig. 5 The interior tower consists of a wooden scaffolding supporting the coelostat assembly. The wooden structure dampens vibrations and is additionally erected on its own foundation. Black apertures prevent stray light from entering the optical path.

\section{Telescope}

The tower telescopes at the Mt. Wilson Observatory in California served as an inspiration for the Einstein Tower (Jäger 1986), which was the first solar tower telescope built in Europe (vertical and horizontal sections of the Einstein Tower are presented in Fig. 3). The telescope is protected by a wooden dome with a diameter of $4.5 \mathrm{~m}$. The wood paneling prevents condensation and the build-up of dew. The exterior of the dome is protected by a sheet metal cladding against the forces of the elements. Two plane coelostat mirrors with an aperture of $60 \mathrm{~cm}$ catch the sunlight at a height of $15 \mathrm{~m}$ above ground and direct the light beam vertically into the tower (Fig. 4). The main mirror has a parallactic mount, which can be rotated in azimuth preserving its parallactic orientation. This prevents vignetting by the shadow casted by the secondary mirror and its mount. Height and tilt of the secondary mirror are adjustable to accommodate the Sun's changing zenith distance over the course of a year and the azimuth angle of the main mirror (north corresponds to the normal position). The original silver-coated $85-\mathrm{cm}$ coelostat mirrors were replaced in the mid-1950ies by $60-\mathrm{cm}$ aluminum-coated glass mirrors. Since 1993 Zerodur mirrors are installed with much improved heat expansion properties minimizing deformations under heat load. Only a single optical element, i.e., a doublet objective lens with a diameter of $D=60 \mathrm{~cm}$ and a focal length of $f \approx 1400 \mathrm{~cm}$, forms a solar image with $d_{\odot} \approx 13 \mathrm{~cm}$ diameter in the optical laboratory, which is located in the basement of the building. Here, another plane folding mirror with a diameter of $45 \mathrm{~cm}$ about $3 \mathrm{~m}$ before the primary focus directs the light beam into the horizontal. The diffraction-limited resolution in the primary focal plane is $\alpha=\lambda / D=0.17^{\prime \prime}$ at $500 \mathrm{~nm}$, and the plate scale is $s=14.73^{\prime \prime} \mathrm{mm}^{-1}$.

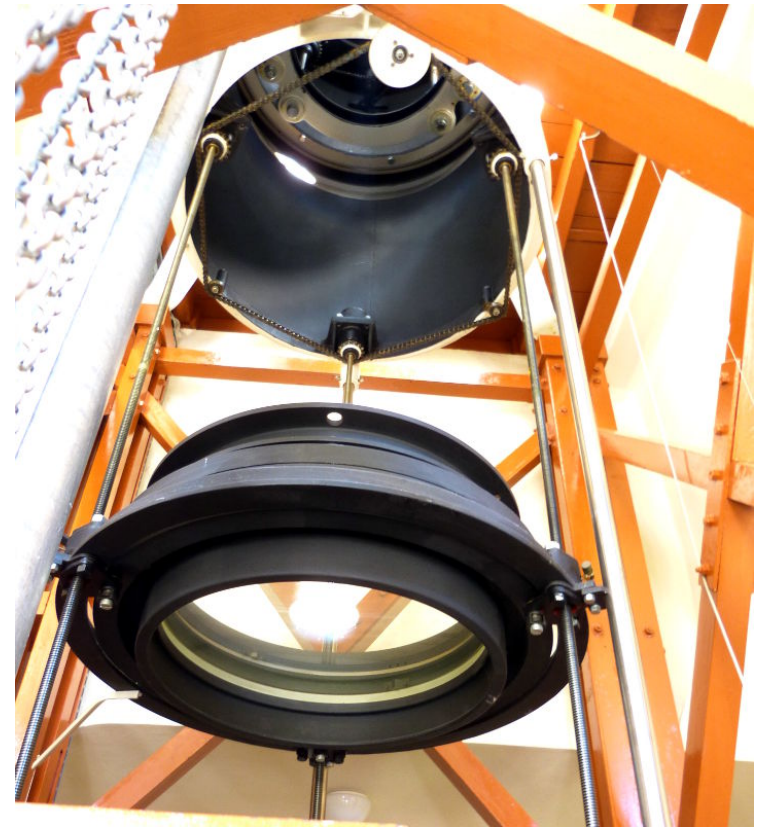

Fig. 6 Mounting of the 60-centimeter doublet lens with gearing mechanism to adjust the focus position.

An aperture stop is situated just below the second coelostat mirror (white donut-shaped object at the bottom of Fig. 4), and it is operated via a pulley from the basement laboratory to select an aperture with a diameter of 10,15 , or $30 \mathrm{~cm}$. Removing the lid with the apertures and pulley from the telescope structure yields the full aperture with a diameter of $60 \mathrm{~cm}$. The smaller apertures are typically used for taking images facilitating higher contrasts when matching seeing- and diffraction-limited resolution. The larger apertures are more suitable for spectroscopic observations, even though highly efficient CCD detectors also deliver good results with the smaller apertures.

The telescope is installed on two stacked wooden towers (Fig. 5) with a separate foundation to insulate the telescope from the wind buffeting the building and vibrations caused by people moving inside the building or outside traffic. Ball and socket joints between foundation, wooden towers, and telescope mount further reduce propagation of vibrations. The low heat expansion coefficient of wood also minimizes thermal expansion and thus focus drifts over the day. The lens mount is attached to three threaded rods for focusing (Fig. 6). The vertical lifting distance is more than a meter in both directions, conveniently placing the focal plane where required for different set-ups in the optical laboratory. This is needed in particular, when inserting the magnification system for direct viewing of the $50-\mathrm{cm}$ diameter solar image.

\subsection{Spectrograph}

The two spectrographs of the Einstein Tower are located in the southern part of the basement, where the upper part (about $1 \mathrm{~m}$, see Fig. 7) of the room is still above ground but 


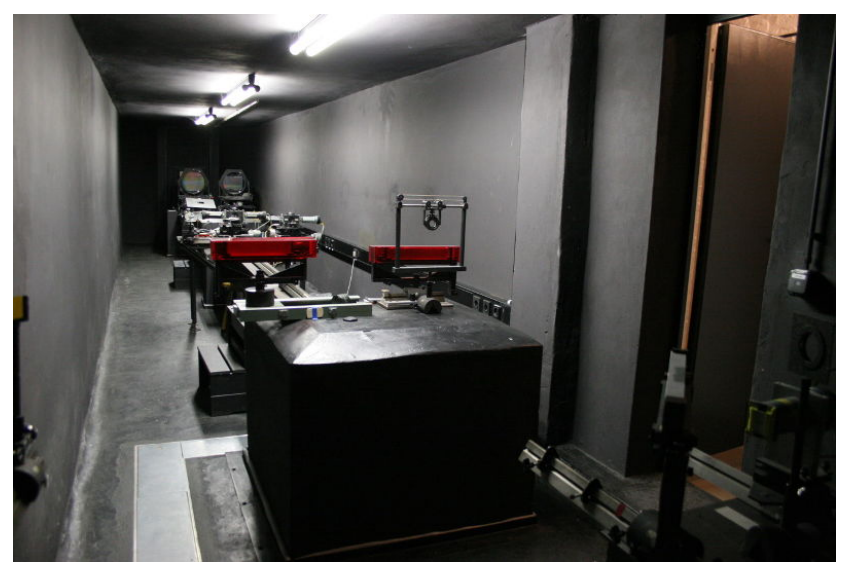

Fig. 7 Interior of the spectrograph chamber with the twin high-resolution spectrographs. Large-format gratings are located in the back behind collimating lenses used in double path. An optical bench on a separate foundation supports the spectrograph optics and facilitates insertion of additional optical elements. Turning mirrors (one in the foreground and two with red protective plastic covers) redirect the light to (polarization) optics or CCD cameras.

below a grass-covered surface (see Figs. 1 and 3). The purpose of the sod is to minimize diurnal temperature changes inside the spectrograph chamber because of its exposed sunlit placement. This technique is nowadays often encountered in green flat-roofed buildings. Another measure to provide a stable thermal environment, which is required for high resolution spectroscopy, is the separate spectrograph chamber inside the basement. The chamber is surrounded on three sides by a corridor, which gives access to camera ports and offers some storage space. The air between interior and exterior walls and above the roof of the spectrograph chamber provides the thermal insulation and acts as a barrier for moisture penetrating the exterior walls. Electric dehumidifiers remove atmospheric moisture and keep the humidity at a constant level in the gallery. The concept of a nested spectrograph chamber was also included in the much more elaborate design of PEPSI (Strassmeier et al. 2015), however, with a much higher stability for temperature and humidity. The interior of the spectrograph chamber is painted in a matt black finish to minimize stray light (see Fig. 7). The spectrographs rest on their own foundation, which is separated from the rest of the building to reduce vibrations and mechanical shocks. A long optical rail, again on its own foundation, is placed in front of the main spectrograph so that polarization optics and measuring devices can be mounted.

The spectrograph chamber houses two high-resolution spectrographs with different gratings. The gratings have dimensions of $420 \mathrm{~mm} \times 320 \mathrm{~mm}$ and $320 \mathrm{~mm} \times 220 \mathrm{~mm}$, respectively. The corresponding grating constants are $1 / d_{1,2}=632$ and 594 lines $\mathrm{mm}^{-1}$ leading to a total number of lines $N_{1,2} \approx 265000$ and 190000 . The spectral resolution is thus $\mathfrak{R}_{1,2}=m N_{1,2} \approx \lambda / \Delta \lambda_{1,2}=1000000$ and 760000 for observations in the fourth order $(m=4)$, which yields $\Delta \lambda_{1,2} \approx 0.59$ and $0.83 \mathrm{pm}$ at an observed wavelength of $\lambda=$ $630.2 \mathrm{~nm}$. The grating equation $m \lambda=d(\sin \alpha+\sin \beta)$, where $\alpha$ and $\beta$ are the angles of incidence and reflection, simplifies in the Littrow configuration of the spectrographs $(\alpha \simeq \beta)$ to $m \lambda=2 d \sin \beta$. Thus, for blaze angles $\beta_{1,2}=52.8^{\circ}$ and $48.5^{\circ}$ the spectral region at $\lambda=630.2 \mathrm{~nm}$ is observed in the fourth order. Each spectrograph uses a $35-\mathrm{cm}$ diameter lens made by Zeiss in double path, i.e., they serve at the same time as collimator and camera lenses. The focal length of the lenses is identical $\left(f_{\mathrm{S}}=1200 \mathrm{~cm}\right)$ and roughly matches the focal length of the telescope $f_{\mathrm{T}}=1400 \mathrm{~cm}$. The linear dispersion for both spectrographs is given as

$$
\left(\frac{\Delta \lambda}{\mathrm{d} x}\right)_{1,2}=\frac{d_{1,2} \cos \beta_{1,2}}{m f_{\mathrm{S}}}=19.9 \text { and } 23.3 \mathrm{pm} \mathrm{mm}^{-1} \text {. }
$$

Since early 2016, an Alta F9000 full frame CCD camera manufactured by Apogee (now part of Andor Technology) was installed at the Einstein Tower replacing outdated CCD cameras. The main area of application is spectropolarimetry using the high-resolution spectrographs. Thus, a large-detector format and a good sensitivity were the selection criteria leading to the acquisition of this camera with a KAF-090000 front-illuminated sensor. The manufacturer specifications (Andor 2016) provide the following characteristics: The detector has $3056 \times 3056$ pixels, and the pixel size is $12 \mu \mathrm{m} \times 12 \mu \mathrm{m}$. Therefore, the total imaging area is $36.7 \mathrm{~mm} \times 36.7 \mathrm{~mm}$. The full well capacity is $94000 \mathrm{e}^{-}$ with a dark current of $0.07 \mathrm{e}^{-}$pixel $^{-1} \mathrm{~s}^{-1}$ and a read noise of $16.1 \mathrm{e}^{-}$at $2.9 \mathrm{MHz}$. The readout is relatively slow with 0.3 frames s $^{-1}$ but still sufficient for spectropolarimetric observations. Thermoelectric in combination with forced air cooling stabilizes the detector temperature to $45{ }^{\circ} \mathrm{C}$ below ambient. The dynamic range is $75.3 \mathrm{~dB}(5840: 1)$ so that the 16-bit digitization is more than required. The spectral coverage of the detector is $360-1080 \mathrm{~nm}$ with a maximum quantum efficiency of $70 \%$ at $580 \mathrm{~nm}$. The quantum efficiency is $34 \%$ at $400 \mathrm{~nm}$, which facilitates observations of the important chromospheric absorption lines $\mathrm{Ca}$ II $\mathrm{H} \& \mathrm{~K}$. In the near-infrared, the quantum efficiency drops to below $10 \%$ at $980 \mathrm{~nm}$. Equation 1 yields a spectral coverage of $0.73 \mathrm{~nm}$ in the order $m=4$ for the $630.2 \mathrm{~nm}$ spectral region and a dispersion of $0.24 \mathrm{pm}_{\text {pixel }}{ }^{-1}$.

Figures 8 and 9 illustrate the performance of telescope and spectrograph, depicting a $\mathrm{NaD}_{1} 589.6 \mathrm{~nm}$ spectrum taken during the Mercury transit on 2016 May 9 and the spectral region around $630.2 \mathrm{~nm}$, which contains two commonly used Fe I and nearby telluric lines. The sunspot spectrum acquired on 2016 April 29 (Fig. 9) clearly shows the Zeeman broadening of the $\mathrm{Fe}_{\mathrm{I}}$ lines, and a selection of quiet Sun, penumbral, and umbral spectral profiles is displayed in the upper panel. To demonstrate the very high resolving power of the spectrograph, a spectrum from the Kitt Peak Fourier Transform Spectral (FTS) atlas (Neckel \& Labs 1984) is shown for comparison. The line widths are almost identical. However, the line depths of the photospheric lines are much deeper for the FTS spectrum because stray 


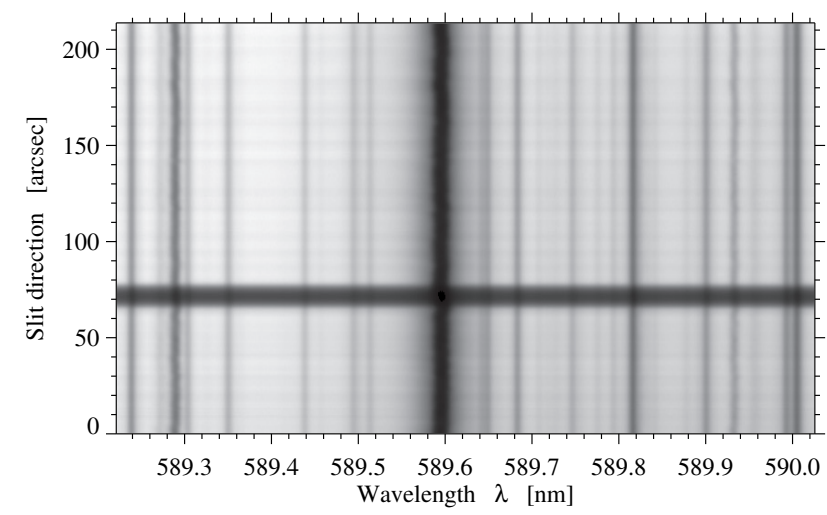

Fig. 8 Spectrum of the chromospheric $\mathrm{NaD}_{1} 589.6 \mathrm{~nm}$ line observed during the Mercury transit on 2016 May 9.
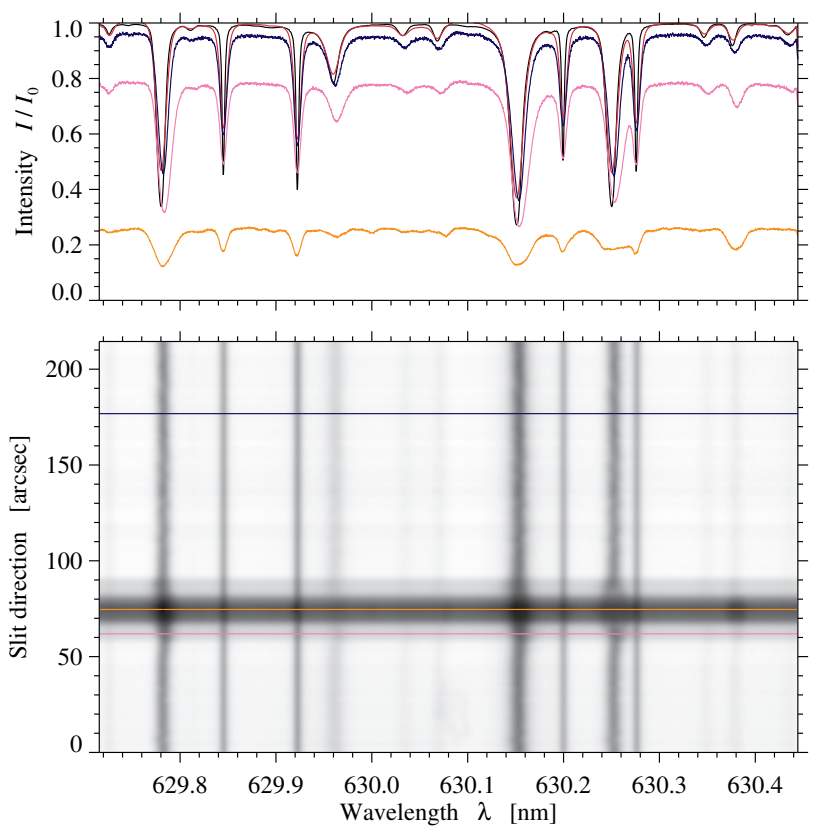

Fig. 9 Sunspot spectrum of the $630.2 \mathrm{~nm}$ spectral region (bottom) observed on 2016 April 29 and selected spectral profiles (top): FTS atlas (black), average quiet-Sun (red), quiet-Sun (blue), penumbra (orange), and umbra (pink).

light contributions are negligible for this type of instrument, in contrast to the Einstein Tower spectra, where significant spectral stray light is present. The strong telluric lines in the observed spectrum are typical for low-altitude sites such as Potsdam.

\subsection{Photographic full-disk observations}

The solar image in the primary focus has a diameter of $d_{\odot} \approx 13 \mathrm{~cm}$ so that direct imaging of the entire solar disk requires a detector of similar size, which is only feasible with photographic plates. For example, the largest monolithic CCD detectors embedded in PEPSI (Strassmeier et al. 2015) have 112 megapixels and a detector size of $95 \mathrm{~mm} \times 95 \mathrm{~mm}$, which would be still too small for the full-disk image. Be-

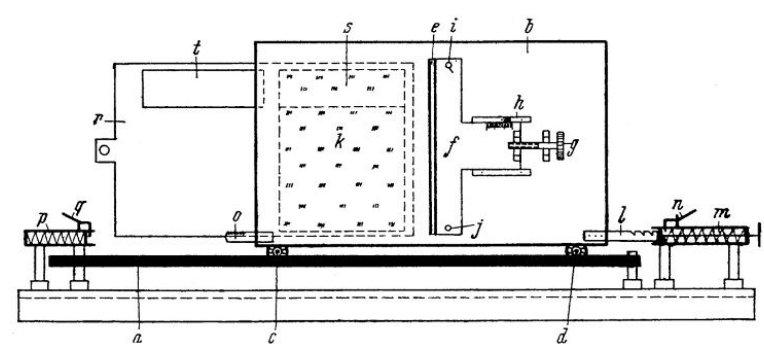

Fig. 10 Schematic sketch of the slit camera for solar fulldisk images: $(a)$ rail of polished round-bar steel, $(b)$ movable rectangular cover plate, $(c, d)$ ball bearing mounted rolls, $(e)$ slit, $(f)$ adjustable slit-jaw, $(g)$ knurled-head screw for precise adjustment of the slit width, $(h)$ scale for the slit width, $(i, j)$ tommy screws for clamping the slit-jaw, (k) photographic plate behind the cover plate, $(l-q)$ springloaded mechanism to release the cover plate for an exposure, and $(r-t)$ devices to add intensity scale and directional marks (see Fig. 2 in von Klüber \& Müller 1948).

fore CCD detectors became available, a photographic camera was required to uniformly illuminate a large-format photographic plate employing short $\left(t_{\exp } \approx 10 \mathrm{~ms}\right)$ exposure times to 'freeze' the seeing. Iris blade shutters are impracticable because of the size of the solar image and the non-uniform illumination pattern. The design and principles of operation of a camera that overcomes these obstacles were described in von Klüber \& Müller (1948), and a schematic sketch of the photographic imaging device is shown in Fig. 10. This camera was in operation until the late 1980ies but does not any longer exist.

A highly polished steel rod with a diameter of $18 \mathrm{~mm}$ serves as the guide rail for the shutter. The rail has to be horizontal and dust free to deliver a uniformly exposed photographic plate. The shutter plate has a vertical slit, which can be adjusted in width to change the exposure time. The large-format photographic plates with dimensions of $18 \mathrm{~cm}$ $\times 24 \mathrm{~cm}$ are mounted in a wooden frame. A spring-loaded mechanism releases the shutter, which then slides rapidly $\left(v=1-2 \mathrm{~m} \mathrm{~s}^{-1}\right)$ past the photographic plate. To keep the speed constant, the approximately 1-meter long rail was tilted by about $2 \mathrm{~mm}$ to compensate friction. The length and tension of spring as well as the length of the holding and release mechanism are critical because the acceleration of the shutter has to stop before the slit reaches the photographic plate so that the motion is uniform. Finally, once the exposure is finished, a catch mechanism with a spring decelerates the shutter and arrests its motion. In a second exposure directional marks and a grayscale are added to determine the terrestrial coordinates and to facilitate photometric analysis of the solar full-disk images. Heliographic coordinates are computed with the help of the coelostat angle and the solar position angle.

About 3000 full-disk images exist in the photographic plate archive of the Einstein Tower covering the period from 1943 to the mid-1980ies. The coverage is usually good but 

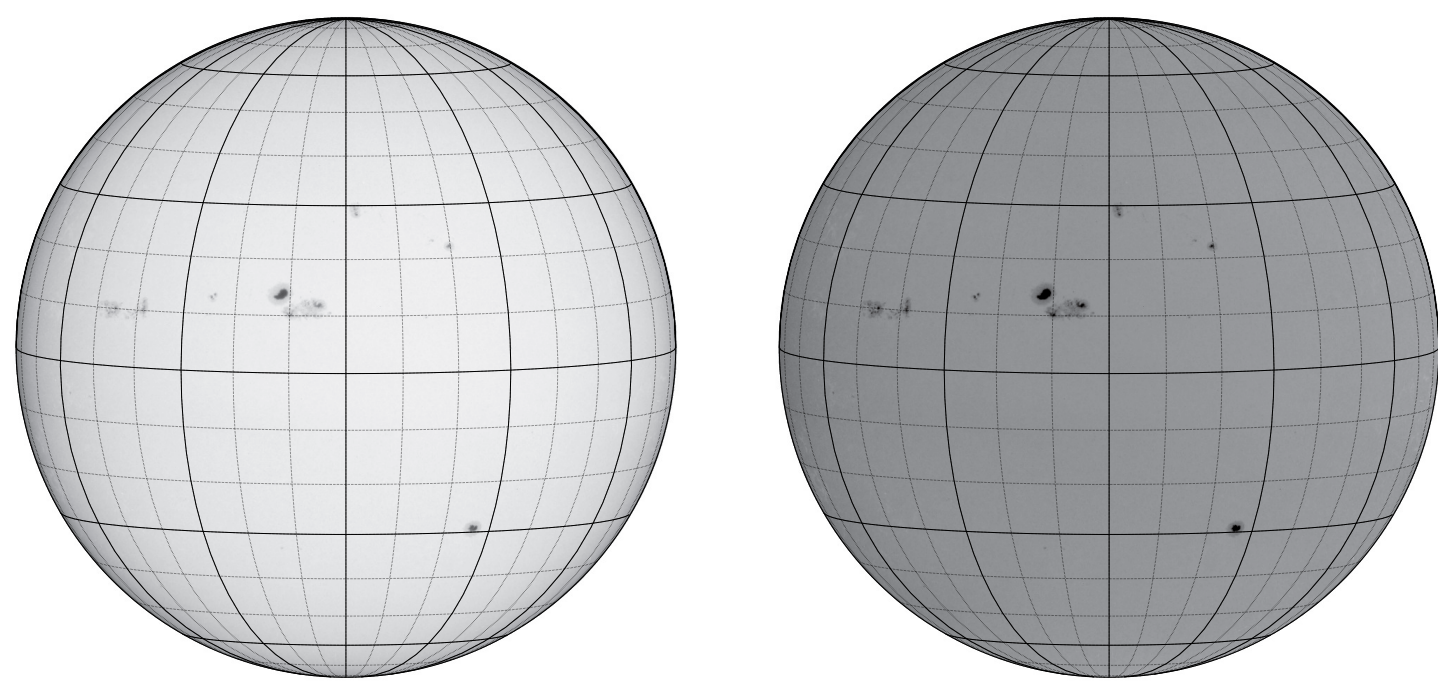

Fig. 11 Solar full-disk image (left) and corresponding limb darkening corrected image (right) observed at the solar observatory Einstein Tower at 07:16 UT on 1947 July 17. The position angle $P=4.3^{\circ}$ is corrected, and heliographic coordinates are indicated in intervals of $30^{\circ}$ (solid lines) and $10^{\circ}$ (dotted lines), respectively.

drops significantly towards the end of this period. These plates were digitized in recent years with the help of highschool students carrying out internships at AIP. The scanned images have $7086 \times 7086$ pixels, and the raw data are saved as monochromatic 8-bit images in the Tagged Image File Format (TIFF). Calibrated and limb-darkening corrected full-disk images are saved as 4-megapixel files in the Flexible Image Transport System (FITS) format. Examples are depicted in Fig. 11, where the limb-darkening was removed using a similar procedure as in Denker et al. (1999). These contrast-enhanced images show besides sunspots and pores also clear signatures of facular regions near the solar limb with good seeing conditions (von Klüber \& Müller 1948).

Solar full-disk images are no longer taken at the Einstein Tower. However, the large format of the Apogee Alta camera covers a field-of-view (FOV) of 530" $\times 530^{\prime \prime}$ in the primary focus, which is sufficient to cover even major active regions. Large-format neutral density and color filters are used to significantly reduce the light level, while still guaranteeing short exposure times $\left(t_{\exp } \approx 100 \mathrm{~ms}\right)$. The image scale of direct solar images is $0.18^{\prime \prime}$ pixel $^{-1}$, which means undersampling by a factor of two, if the full telescope aperture is used. However, considering the seeing conditions at Potsdam, achieving diffraction-limited observations even with image restoration techniques provides a formidable challenge. On the other hand, the observing conditions are sufficiently good to detect facular regions near the solar limb and sunspot fine-structure in solar full-disk images (see right panel of Fig. 11).

\section{Research and development}

Many research and development projects have benefited from the light-gathering power of the $60-\mathrm{cm}$ diameter solar telescope and the stable thermal environment of the spectrograph chamber. A major advantage offered by the Einstein Tower is its availability for long-term measurements and equipment tests. Optimization of and adjustments to the instrument design can thus easily be made at the institute's nearby workshops. As a consequence, mature instruments can be delivered to world-class telescopes, where telescope time is at a premium.

Components of the Stokes-IQUV spectropolarimeter (Ilyin et al. 2011; Strassmeier et al. 2003) for the Potsdam Échelle Polarimetric and Spectroscopic Instrument (PEPSI, Strassmeier et al. 2015, 2008) were tested in the optical laboratory of the Einstein Tower before delivering the spectropolarimeter to the Large Binocular Telescope (LBT) at Mt. Graham International Observatory in Arizona. PEPSI resides at the LBT's Gregorian focus station and achieves a spectral resolution of $\mathcal{R} \approx 120000$ in the polarimetric mode, which is available for the wavelength range $383-907 \mathrm{~nm}$ using three exposures for full spectral coverage. The lightgathering power of the $2 \times 8.4$-meter aperture LBT enables a polarimetric accuracy of up to $10^{-4}$ for stellar observations previously only available for solar spectropolarimetry. The tested opto-mechanical components included rotary stages holding the polarimetric calibration optics, which consists of a Glan-Thompson prism and two quarter-wave plates. In addition, a modified Foster prism beam-splitter, serving as a linear polarizer, was evaluated.

The altitude-azimuth mount (Volkmer et al. 2012) of the 1.5-meter aperture GREGOR solar telescope (Schmidt et al. 2012) leads to a complex optical path traversing elevation and declination axes as well as the image rotator and the GREGOR Adaptive Optics System (GAOS, Berkefeld et al. 2012). Cover windows of the partially evacuated light path and a total of 16 mirrors introduce a time dependent 


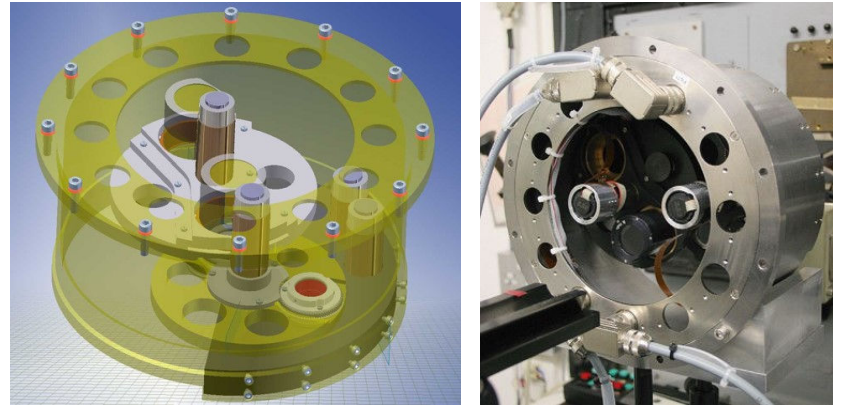

Fig. 12 Construction model of the GPU (left) and the GPU mounted on the optical in front of the spectrograph entrance slit during calibration measurements (right).

instrumental polarization. The GREGOR Polarimetric Unit (GPU, Balthasar et al. 2011; Hofmann et al. 2012) is located at the telescope's secondary focus, where the FOV is already significantly reduced, and the beam diameter is sufficiently small to accommodate the available size of the polarization calibration optics. The optical elements are mounted on two motorized wheels and motorized rotation stages are used to position a linear polarizer and two quarter wave retarders for the visible and near-infrared. The GPU is located still in the symmetric part of the optical train before the Nasmyth mirror, which directs the light into the coudé optical train. Thus, the instrumental polarization of the coudé optical train, image rotator, and AO system is calibrated. A construction drawing of the GPU is shown in the left panel of Fig. 12.

The characteristics of the polarizers and retarders were determined in the optical laboratory of the Einstein Tower (Hofmann et al. 2009), where the GPU was placed in front of the spectrograph entrance (see right panel of Fig. 12). An air-spaced Marple-Hess prism (a particular form of a double Glan prism) has to withstand an extreme power density of $25 \mathrm{~W} \mathrm{~cm}^{-2}$ and has to achieve an extinction ratio of $10^{-5}-$ $10^{-6}$. The measurements showed that a retardation accuracy of $\lambda / 100$ and a positioning accuracy of $0.1^{\circ}$ yields Müller matrices with an accuracy at the $10^{-4}$ level (Hofmann 2008). The measured extinction ratio was $2 \times 10^{-6}$, and the angular position of the intensity minimum was independent of the wavelength range $(400-1000 \mathrm{~nm})$ within the measurement errors. The fast axis of combined zero-order retarders is a function of wavelength, which was determined across the visible $(379-980 \mathrm{~nm})$ and near-infrared $(656-1105 \mathrm{~nm})$ wavelength ranges. Finally, the tests demonstrated that the broad- and narrow-band efficiency of the GPU to generate circular polarized light is very close to unity across the desired wavelength range and matches the design specifications (Hofmann et al. 2009).

Solar Orbiter (Müller et al. 2013) is an ESA mission to be launched in October 2018 and serves a platform for multiple instruments including the Spectrometer/Telescope for Imaging X-rays (STIX, Benz et al. 2012). Initial tests, optimization, and calibration of the STIX Aspect System (SAS) were performed at the Einstein Tower before test and verifi-

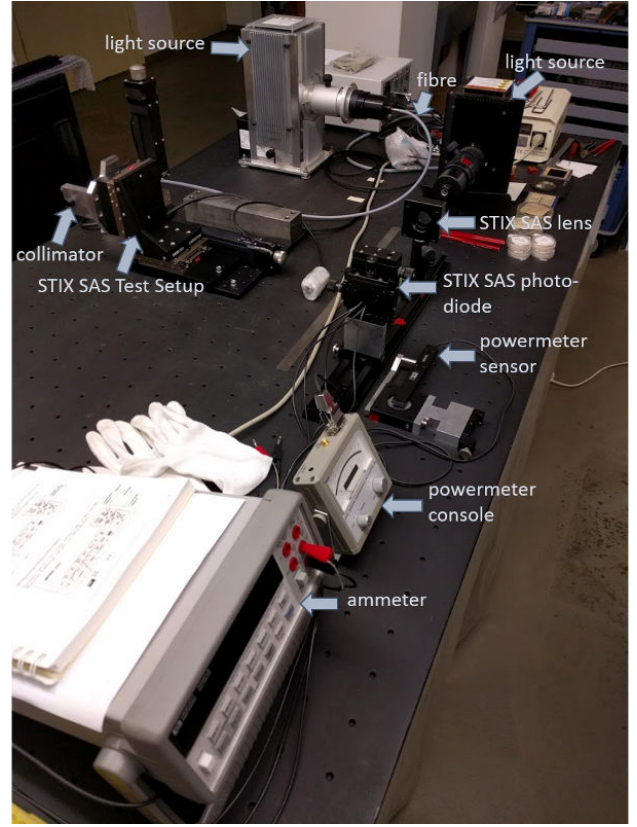

Fig. 13 Optical table with two set-ups for STIX: SAS simulator to calibrate the STIX lens and photodiode (right) and SAS verification system of STIX flight and flight spare models (left).

cation campaigns are carried out with the flight and flight spare models. To improve the pointing of the spacecraft, SAS functions as a solar limb detector and provides very accurate positioning for STIX (few seconds of arc). This facilitates coordinated observing campaigns with other groundbased or spaceborne instruments (Krucker et al. 2013). An SAS simulator will be installed in the optical laboratory to investigate and verify the SAS data obtained during all phases of the Solar Orbiter mission. Some of the equipment (see Fig. 13) was tested in the optical laboratory of the Einstein Tower.

Besides research and development in solar instrumentation, other projects have exploited the unique characteristics of the Einstein Tower's spectrograph optics and the stable thermal environment of the subterranean spectrograph chamber. Advanced cementing technology was tested for a $20-\mathrm{cm}$ diameter open reflector with plane mirrors (Neubert et al. 2012), which will be used in laser communications with low earth orbit (LEO) satellites. Using the large, $35-\mathrm{cm}$ diameter collimator lens of the spectrograph, the assembly and manufacturing accuracy was validated (nearing the $0.2^{\prime \prime}$ target), gravity-induced distortions in different mounting positions were identified, and long-term drifts were measured for the triple mirror assembly.

\section{Education and public outreach}

The Science Park Albert Einstein with its ensemble of historical science buildings in an English landscape garden is open to the public during daytime hours. It is home to the 
German Research Center for Geosciences (GFZ) and the Potsdam Institute for Climate Impact Research (PIK). In addition, remote stations of the Alfred Wegener Institute for Polar and Marine Research (AWI), the German Meteorological Office (DWD), and AIP are located at the Telegrafenberg. The annual "Long Night of the Science" is the main public outreach event, which attracts several thousands of visitors from the Berlin-Potsdam metropolitan area. Many smaller events throughout the year and guided tours for school classes and university courses complement this main event. Solar observations with the Einstein Tower are part of the astronomy and astrophysics curriculum for master students at the University of Potsdam. Experiments include measuring the magnetic field of sunspots and determining the solar differential rotation. Training in spectropolarimetric techniques is also offered to junior scientists at AIP, who typically do not have direct access to and hands-on experience with instruments at today's major astronomical research infrastructures.

\section{Conclusions}

First observations started at the Einstein Tower in 1924 December 6. More than 90 years later the telescope and its instrumentation are still an important asset in AIP's research portfolio. Nowadays, activities are focused on (1) education, training, and public outreach and (2) research and development. Gratifyingly, the upgraded CCD camera system and investments improving positioning of the telescope and tracking active regions will facilitate new science observations. With the second generation instrumentation of GREGOR and the European Solar Telescope (EST, Collados et al. 2010) on the horizon, we expect that the Einstein Tower will play an important role in the related research and development activities.

Acknowledgements. SJGM is grateful for financial support from the Leibniz Graduate School for Quantitative Spectroscopy in Astrophysics, a joint project of the Leibniz Institute for Astrophysics Potsdam and the Institute of Physics and Astronomy of the University of Potsdam. The major restoration of the Einstein Tower in the years 1997-1999 was made possible by generous funds of the Wüstenrot Foundation. Ongoing conservation and maintenance of the Einstein Tower is supported by the State of Brandenburg through institutional and extraordinary funding. The Einstein Tower received in 2015 a planing and conservation grant of the Getty Foundation's "Keeping It Modern" initiative exploring options for preserving the complex and challenging building structure.

\section{References}

Andor 2016, Alta F9000 - High Performance Cooled CCD Camera System, Belfast

Balthasar, H. \& Artus, H. 2000, Sterne und Weltraum, 8, 634

Balthasar, H., Bello González, N., Collados, M., et al. 2011, in ASP Conf. Ser., Vol. 437, Solar Polarization 6, ed. J. R. Kuhn, D. M. Harrington, H. Lin, S. V. Berdyugina, J. Trujillo-Bueno, S. L. Keil, \& T. Rimmele, 351-358
Benz, A. O., Krucker, S., Hurford, G. J., et al. 2012, in Proc. SPIE, Vol. 8443, Space Telescopes and Instrumentation 2012: Ultraviolet to Gamma Ray, 84433L

Berkefeld, T., Schmidt, D., Soltau, D., von der Lühe, O., \& Heidecke, F. 2012, AN, 333, 863

Collados, M., Bettonvil, F., Cavaller, L., et al. 2010, AN, 331, 615

Denker, C., Johannesson, A., Marquette, W., et al. 1999, SoPh, 184, 87

Eggers, B. et al. 1995, Der Einsteinturm in Potsdam (Ars Nicolai, Berlin)

Freundlich, E. F. 1927, Das Turmteleskop der Einstein-Stiftung (Springer, Berlin)

Hentschel, K. 1992, Der Einstein-Turm (Spektrum Akademischer Verlag, Heidelberg)

Hofmann, A. 2008, Centr. Eur. Astrophys. Bull., 32, 17

Hofmann, A., Arlt, K., Balthasar, H., et al. 2012, AN, 333, 854

Hofmann, A., Rendtel, J., \& Arlt, K. 2009, Centr. Eur. Astrophys. Bull., 33, 317

Ilyin, I., Strassmeier, K. G., Woche, M., Dionies, F., \& Di Varano, I. 2011, AN, 332, 753

Jäger, F. W. 1986, Die Sterne, 62, 14

Krucker, S., Benz, A. O., Hurford, G. J., et al. 2013, Nucl. Instr. Meth. Phys. Res. A, 732, 295

Müller, D., Marsden, R. G., St. Cyr, O. C., \& Gilbert, H. R. 2013, SoPh, 285, 25

Neckel, H. \& Labs, D. 1984, SoPh, 90, 205

Neubert, R., Grunwaldt, L., Hamdorf, A., et al. 2012, in Satellite, Lunar and Planetary Laser Ranging: Characterizing the Space Segment, International Technical Laser Workshop, Frascati (Rome), Italy

Pitz \& Hoh 1996, Der Einsteinturm in Potsdam - Voruntersuchung zur Sanierung, Werkstatt für Architektur und Denkmalpflege, Berlin

Pitz \& Hoh 2006a, Der Einsteinturm in Potsdam - Dokumentation der Instandsetzung, Werkstatt für Architektur und Denkmalpflege, Berlin

Pitz \& Hoh 2006b, Der Einsteinturm in Potsdam - Pflegeplan, Werkstatt für Architektur und Denkmalpflege, Berlin

Sailer, M. P. 2007, Acta Hist. Astron., Vol. 31, Kommandosache Sonnengott. Geschichte der deutschen Sonnenforschung im Dritten Reich und unter alliierter Besatzung (Verlag Harri Deutsch, Frankfurt am Main)

Schmidt, W., von der Lühe, O., Volkmer, R., et al. 2012, AN, 333, 796

Staude, J. 1991, Sterne und Weltraum, 30, 505

Staude, J. 1995, Die Sterne, 71, 142

Strassmeier, K. G., Hofmann, A., Woche, M. F., et al. 2003, in Proc. SPIE, Vol. 4843, Polarimetry in Astronomy, ed. S. Fineschi, 180-189

Strassmeier, K. G., Ilyin, I., Järvinen, A., et al. 2015, AN, 336, 324

Strassmeier, K. G., Woche, M., Ilyin, I., et al. 2008, in Proc. SPIE, Vol. 7014, Ground-Based and Airborne Instrumentation for Astronommy, ed. I. S. McLean \& M. M. Casali, 70140N

Volkmer, R., Eisenträger, P., Emde, P., et al. 2012, AN, 333, 816

von Klüber, H. \& Müller, H. 1948, Z. Astrophys., 24, 207, Mitt. Astrophys. Obs. Potsdam Nr. 20

Wilderotter, H. 2005, Ein Turm für Albert Einstein. Potsdam, das Licht und die Erforschung des Himmels (L\&H Verlag, Hamburg) 